\title{
Stochastic Cooling with Schottky Band Overlap*
}

\author{
Valeri Lebedev
}

Fermilab, P.O. Box 500, Batavia, IL 60510

\begin{abstract}
Optimal use of stochastic cooling is essential to maximize the antiproton stacking rate for Tevatron Run II. Good understanding and characterization of the cooling is important for the optimization. The paper is devoted to derivation of the Fokker-Planck equations justified in the case of near or full Schottky base overlap for both longitudinal and transverse coolings.
\end{abstract}

Keywords: Stochastic cooling, band overlap, antiproton stacking.

PACS: 29.20.-C.

\section{INTRODUCTION}

The Schottky band overlap compromises the performance of stochastic cooling. Therefore all practical cooling systems are designed and built to avoid the band overlap. Nevertheless, operating cooling systems are frequently used in a regime when bands are close to overlap or slightly overlapped. In this case the band overlap needs to be taken into account if detailed description of the cooling is required. The stochastic cooling theory with no band overlap is well developed [see Ref. 1 and 2 and included bibliography]. In this paper we extend this theory to the case of arbitrary band overlap. First, we derive expressions for the beam permeabilities of the longitudinal and transverse coolings and, then, proceed to derivation of the FokkerPlanck equations describing transverse and longitudinal coolings.

\section{BEAM PERMEABILITY FOR LONGITUDINAL COOLING}

Usually, a calculation of the beam permeability is based on azimuthal harmonics. It does not work well if bands are close being overlapped because the amplitudes of the harmonics are changed within one revolution. In this paper we limit ourselves to the case of the beam with sufficiently small intensity so that the beam interaction with vacuum chamber could be neglected. That allows us to reduce the problem from one of finding the entire ring distribution function to one of finding the local distribution functions in the pickup and kicker. Let $f_{1}(x, t)$ be the distribution function immediately after the kicker, $f_{2}(x, t)$ be the distribution function in the pickup, and $f_{3}(x, t)$ be the distribution function just before the kicker. Taking into account that the particle momentum is changed only in the kicker one can write the equations binding up these functions:

* Work supported by the Universities Research Assos., Inc., under contract DE-AC02-76CH03000 with the U.S. Dept. of Energy. 


$$
\begin{aligned}
& f_{2}(x, t)=f_{1}\left(x, t-T_{1}\left(1+\eta_{1} x_{0}\right)\right), \\
& f_{3}(x, t)=f_{2}\left(x, t-T_{2}\left(1+\eta_{2} x\right)\right), \\
& \left.f_{1}(x, t)=f_{3}\left(x-\delta p(t) / p_{0}, t\right)\right) .
\end{aligned}
$$

Here $x=\left(p-p_{0}\right) / p_{0}$ is the relative momentum deviation, $T_{1}, T_{2}$ and $T_{0}=T_{1}+T_{2}$ are the kicker-to-pickup, pickup-to-kicker and revolution times for the reference particle, $\eta=\alpha-1 / \gamma^{2}$ is the slip factor, $\eta_{1}$ and $\eta_{2}$ are the partial kicker-to-pickup and pickupto-kicker slip factors so that $\eta T_{0}=\eta_{1} T_{1}+\eta_{2} T_{2}$, and $\delta p(t)$ is the particle momentum change by the kicker. Expressing the distribution function through its equilibrium value and the perturbation, $f_{k}(x, t)=f_{0}(x)+\tilde{f}_{k}(x, t), k=1, \ldots 3$, and leaving only the first order addend in the Taylor expansion of the third equation in Eq. (1) one obtains:

$$
\begin{aligned}
& \tilde{f}_{2}(x, t)=\tilde{f}_{1}\left(x, t-T_{1}\left(1+\eta_{1} x_{0}\right)\right), \\
& \tilde{f}_{3}(x, t)=\tilde{f}_{2}\left(x, t-T_{2}\left(1+\eta_{2} x\right)\right), \\
& \tilde{f}_{1}(x, t)=\tilde{f}_{3}(x, t)-\frac{\delta p(t)}{p_{0}} \frac{d f_{0}(x)}{d x} .
\end{aligned}
$$

Looking for a solution in the form $\tilde{f}_{k}(x, t)=\tilde{f}_{k \omega}(x) e^{i \omega t}, \delta p(t)=\delta p_{\omega} e^{i \omega t}$ and excluding $\tilde{f}_{1 \omega}(x)$ and $\tilde{f}_{3 \omega}(x)$ from the resulting equations we obtain:

$$
\tilde{f}_{2 \omega}(x) \exp \left(i \omega T_{1}\left(1+\eta_{1} x\right)=\tilde{f}_{2 \omega}(x) \exp \left(-i \omega T_{2}\left(1+\eta_{2} x\right)-\frac{d f_{0}(x)}{d x} \frac{\delta p_{\omega}}{p_{0}} .\right.\right.
$$

Let the momentum kick be determined by the sum of amplified pickup signal and an external harmonic perturbation so that:

$$
\delta p_{\omega} / p_{0}=\int d x \tilde{f}_{2 \omega}(x) G(x, \omega) e^{-i \omega T_{2}}\left[1-A(\omega) e^{-i \omega T_{0}}\right]+\Delta p_{\text {ext } \omega} / p_{0} .
$$

Here the term $e^{-i \omega T_{2}}$ takes into account the delay in signal propagation from the pickup to the kicker, $\tilde{f}_{2}\left(p, t-T_{2}\right) \rightarrow \tilde{f}_{2 \omega}(p) e^{-i \omega T_{2}}$. The total system gain, $G(x, \omega)\left(1-A(\omega) e^{-i \omega T_{0}}\right]$, is chosen so that it would describe both Palmer and momentum cooling. For Palmer cooling $A(\omega)=0$ and the pickup signal depends on the particle momentum due to nonzero dispersion in the pickup. For filter cooling the pickup signal does not depend on particle momentum, $G(x, \omega) \rightarrow G(\omega)$, and the cooling signal is formed by the notch filter, $A(\omega) \approx 1$. Its delay is equal to the revolution time for the reference particle, $T_{0}$. Taking into account the distribution function normalization, $\int f_{0}(x) d x=1$, and introducing the impedances of pickup, $Z_{p}$, and kicker, $Z_{k}$, so that the pickup voltage is

$$
U_{\text {pickup }_{\omega}}=I_{0} \int Z_{p}(x, \omega) f_{2 \omega}(x, \omega) d x,
$$

and the energy gain in the kicker is

$$
\delta E_{\text {kicker }_{o}}=e \frac{Z_{k}(\omega)}{Z_{\text {ampl }}} U_{\text {kicker }_{o}},
$$

we obtain that the system gain is:

$$
G(x, \omega)=\frac{e I_{0} Z_{p}(x, \omega) Z_{k}(\omega)}{\gamma \beta^{2} m c^{2} Z_{\text {ampl }}} K(\omega) .
$$


Here $I_{0}$ is the beam current, $Z_{\text {ampl }}=50 \Omega$ is the impedance of power amplifier, $K(\omega)$ is the total electronic amplification of the cooling system, $c$ is the speed of the light, $e$ and $m$ are the particle charge and mass, and $\beta$ and $\gamma$ are the relativistic factors.

Substitution Eq. (4) into Eq. (3) yields:

$$
\begin{aligned}
& \tilde{f}_{2 \omega}(x)\left[e^{i \omega T_{1}\left(1+\eta_{1} x\right)}-e^{-i \omega T_{2}\left(1+\eta_{2} x\right)}\right]+ \\
& \quad \frac{d f_{0}(x)}{d x}\left[\frac{\Delta p_{e x t \omega}}{p_{0}}+e^{-i \omega T_{2}}\left[1-A(\omega) e^{-i \omega T_{0}}\right] \int d x^{\prime} \tilde{f}_{2 \omega}\left(x^{\prime}\right) G\left(x^{\prime}, \omega\right)\right]=0 .
\end{aligned}
$$

Dividing both addends by $e^{i \omega T_{1}\left(1+\eta_{1} x\right)}-e^{-i \omega T_{2}\left(1+\eta_{2} x\right)}$, multiplying them by $G(x, \omega)$ and integrating we obtain:

$$
\begin{aligned}
S_{\omega}+ & \frac{\Delta p_{\text {ext } \omega}}{p_{0}} \int \frac{d f_{0}(x)}{d x} \frac{G(x, \omega) d x}{e^{i \omega T_{1}\left(1+\eta_{1} x\right)}-e^{-i \omega T_{2}\left(1+\eta_{2} x\right)}}+ \\
& e^{-i \omega T_{2}}\left[1-A(\omega) e^{-i \omega T_{0}}\right] S_{\omega} \int \frac{d f_{0}(x)}{d x} \frac{G(x, \omega) d x}{e^{i \omega T_{1}\left(1+\eta_{1} x\right)}-e^{-i \omega T_{2}\left(1+\eta_{2} x\right)}}=0,
\end{aligned}
$$

where $S_{\omega}=\int d x^{\prime} \tilde{f}_{2 \omega}\left(x^{\prime}\right) G\left(x^{\prime}, \omega\right)$. Solving Eq. (9) relative to $S_{\omega}$ we finally obtain the system response at the pickup location due to the external harmonic perturbation:

$$
S_{\omega}=-\frac{1}{\varepsilon(\omega)} \frac{\Delta p_{e x t \omega}}{p_{0}} \int_{\delta \rightarrow 0_{+}} \frac{d f_{0}(x)}{d x} \frac{G\left(x^{\prime}, \omega\right) e^{i \omega T_{2}\left(1+\eta_{2} x\right)}}{e^{i \omega T_{0}(1+\eta x)}-(1-\delta)} d x,
$$

where $\varepsilon(\omega)$ is the beam permeability

$$
\varepsilon(\omega)=1+\left(1-A(\omega) e^{-i \omega T_{0}}\right) \int_{\delta \rightarrow 0_{+}} \frac{d f_{0}(x)}{d x} \frac{G(x, \omega) e^{i \omega T_{2} \eta_{2} x}}{e^{i \omega T_{0}(1+\eta x)}-(1-\delta)} d x
$$

In the above equations the rule to traverse the poles, $\delta \rightarrow 0_{+}$, follows from the fact that for the complex Laplace transform $\omega$ is shifted to the lower complex plane.

Far away from Schottky band overlap the exponent in the denominator of Eq. (11) can be expanded near revolution harmonic, $\omega=n \omega_{0}+\delta \omega, \omega_{0}=2 \pi / T_{0}$ and we arrive to the standard formula for the permeability ${ }^{[1]}$.

\section{BEAM PERMEABILITY FOR TRANSVERSE COOLING}

Similar to the method used above for the longitudinal cooling the beam evolution is considered at three points: (1) after kicker, (2) in the pickup, and (3) before the kicker. The beam dipole moment at each point is

$$
d_{k}(t)=\frac{I_{0}}{c \beta} \int y_{k}(x, t) f_{0}(x) d x \quad, \quad k=1,2,3 .
$$

Here $f_{0}(x)$ is the distribution function over momentum, and $y_{k}(x)$ is the average transverse beam displacement for particles with relative momentum deviations equal to $x$. Normalizing the beam displacements, $y_{k}(x)$, and angles, $\theta_{k}(x)$, by the beta-

functions so that $\tilde{y}_{k}=y_{k} / \sqrt{\beta_{k}}$ and $\tilde{\theta}_{k}=\theta_{k} \sqrt{\beta_{k}}+\alpha_{k} x_{k} / \sqrt{\beta_{k}}$ one can write the system of equations binding up the beam displacements after and before the kicker: 


$$
\begin{aligned}
& \tilde{y}_{3}(x, t)=c(x) \tilde{y}_{1}\left(x, t-T_{0}(1+\eta x)\right)+s(x) \tilde{\theta}_{1}\left(x, t-T_{0}(1+\eta x)\right), \\
& \tilde{\theta}_{3}(x, t)=-s(x) \tilde{y}_{1}\left(x, t-T_{0}(1+\eta x)\right)+c(x) \tilde{\theta}_{1}\left(x, t-T_{0}(1+\eta x)\right) .
\end{aligned}
$$

Here $c(x)=\cos (2 \pi(v+\xi x)), s(x)=\sin (2 \pi(v+\xi x)), v$ is the betatron tune, and $\xi$ is the tune chromaticity. Passing the kicker changes the beam angle but does not change beam coordinate so that

$$
\begin{aligned}
& \tilde{y}_{1}(x, t)=\tilde{y}_{3}(x, t), \\
& \tilde{\theta}_{1}(x, t)=\tilde{\theta}_{3}(x, t)+\delta \tilde{\theta}(t) .
\end{aligned}
$$

We look for a solution in the form $\tilde{y}_{k}(x, t)=\tilde{y}_{k \omega}(x) e^{i \omega t}$ and $\delta \theta(t)=\delta \theta_{\omega} e^{i \omega t}$. Substituting it into Eqs. (13) and (14) and solving obtained equations relative to $\tilde{y}_{1 \omega}(x)$ and $\tilde{\theta}_{1 \omega}(x)$ we obtain

$$
\begin{aligned}
& \tilde{\theta}_{1 \omega}(x)=-\frac{\left(c(x)-\exp \left(i \omega T_{0}(1+\eta x)\right)\right) \exp \left(i \omega T_{0}(1+\eta x)\right)}{\exp \left(2 i \omega T_{0}(1+\eta x)\right)-2 c(x) \exp \left(i \omega T_{0}(1+\eta x)\right)+1} \delta \tilde{\theta}_{\omega}, \\
& \tilde{y}_{1 \omega}(x)=\frac{s(x) \exp \left(i \omega T_{0}(1+\eta x)\right)}{\exp \left(2 i \omega T_{0}(1+\eta x)\right)-2 c(x) \exp \left(i \omega T_{0}(1+\eta x)\right)+1} \delta \tilde{\theta}_{\omega} .
\end{aligned}
$$

Taking into account the relationship between coordinates and angles of points 1 and 2,

$$
\begin{aligned}
& \tilde{y}_{2}(x, t)=c_{1}(x) \tilde{y}_{1}\left(x, t-T_{1}\left(1+\eta_{1} x\right)\right)+s_{1}(x) \tilde{\theta}_{1}\left(x, t-T_{1}\left(1+\eta_{1} x\right)\right), \\
& \tilde{\theta}_{2}(x, t)=-s_{1}(x) \tilde{y}_{1}\left(x, t-T_{1}\left(1+\eta_{1} x\right)\right)+c_{1}(x) \tilde{\theta}_{1}\left(x, t-T_{1}\left(1+\eta_{1} x\right)\right),
\end{aligned}
$$

and transforming the time dependent values in Eq. (16) to their Fourier harmonics we obtain for the beam displacement in the pickup

$$
\tilde{y}_{2 \omega}(x)=\frac{\left(s_{2}(x)+s_{1}(x) e^{i \omega T_{0}(1+\eta x)}\right) e^{i \omega T_{2}\left(1+\eta_{2} x\right)}}{e^{2 i \omega T_{0}(1+\eta x)}-2 c(x) e^{i \omega T_{0}(1+\eta x)}+1} \delta \tilde{\theta}_{\omega} .
$$

Here $c_{1,2}(x)=\cos \left(2 \pi\left(v_{1,2}+\xi_{1,2} x\right)\right), s_{1,2}(x)=\sin \left(2 \pi\left(v_{1,2}+\xi_{1,2} x\right)\right), 2 \pi v_{1}$ and $2 \pi v_{2}$ are the betatron phase advances between pickup and kicker so that $v_{1}+v_{2}=v$, and $\xi_{1}$ and $\xi_{2}$ are the partial tune chromaticities so that $\xi_{1}+\xi_{2}=\xi$.

Similar to Eq. (4) the beam kick is determined by the sum of amplified pickup signal and an external harmonic perturbation so that:

$$
\delta \tilde{\theta}_{\omega}=\int d x f_{0}(x) \tilde{y}_{2 \omega}(x) G_{\perp}(\omega) e^{-i \omega T_{2}}+\Delta \tilde{\theta}_{\text {ext } \omega} .
$$

We introduce the impedances of pickup, $Z_{p \perp}$, and kicker, $Z_{k \perp}$, so that the pickup voltage is

$$
U_{\text {pickup }_{\omega}}=I_{0} Z_{p \perp}(\omega) \overline{y_{\omega}}=I_{0} Z_{p \perp}(\omega) \int y_{\omega}(x) f_{0}(x) d x,
$$

and the transverse angle obtained by a particle in the kicker is

$$
\delta \theta_{\text {kic ker }_{\omega}}=\frac{e}{m c^{2} \gamma \beta^{2}} \frac{Z_{k \perp}(\omega)}{Z_{\text {ampl }}} U_{\text {kicker }_{\omega}} .
$$

That yields that the system gain is:

$$
G_{\perp}(\omega)=\frac{e I_{0} Z_{p \perp}(\omega) Z_{k \perp}(\omega)}{\gamma \beta^{2} m c^{2} Z_{\text {ampl }}} \sqrt{\beta_{p} \beta_{k}} K(\omega),
$$

where $\beta_{p}$ and $\beta_{k}$ are the beta-functions in the pickup and kicker. 


$$
\begin{aligned}
& \text { Substituting Eq. (18) into Eq. (17) we obtain: } \\
& \tilde{y}_{2 \omega}(x)=\frac{\left(s_{2}(x)+s_{1}(x) e^{2 i \omega T_{0}(1+\eta x)}\right) e^{i \omega T_{2}\left(1+\eta_{2} x\right)}}{e^{2 i \omega T_{0}(1+\eta x)}-2 c(x) e^{i \omega T_{0}(1+\eta x)}+1}\left(G_{\perp}(\omega) e^{-i \omega T_{2}} \int d x f_{0}(x) \tilde{y}_{2 \omega}(x)+\Delta \tilde{\theta}_{e x t \omega}\right) .
\end{aligned}
$$

The solution is similar to the solution carried out in the previous section. The result is:

$$
\overline{\tilde{y}_{2 \omega}} \equiv \int d x f_{0}(x) \tilde{y}_{2 \omega}(x)=\frac{\Delta \tilde{\theta}_{e x t \omega}}{\varepsilon_{\perp}(\omega)} \int d x f_{0}(x) \frac{\left(s_{2}(x)+s_{1}(x) e^{i \omega T_{0}(1+\eta x)}\right) e^{i \omega T_{2}\left(1+\eta_{2} x\right)}}{e^{2 i \omega T_{0}(1+\eta x)}-2 c(x) e^{i \omega T_{0}(1+\eta x)}+1} .
$$

where the beam permeability is:

$$
\varepsilon_{\perp}(\omega)=1-\frac{G_{\perp}(\omega)}{2} \int_{\delta \rightarrow 0_{+}} \frac{\left\lfloor e^{-i \omega T_{0}(1+\eta x)} \sin \left(2 \pi\left(v_{2}+\xi_{2} x\right)\right)+\sin \left(2 \pi\left(v_{1}+\xi_{1} x\right)\right)\right] e^{i \omega T_{2} \eta_{2} x}}{\cos \left(\omega T_{0}(1+\eta x)\right)-\cos (2 \pi(v+\xi x))+i \delta \sin \left(\omega T_{0}(1+\eta x)\right)} f_{0}(x) d x
$$

\section{FOKKER-PLANCK EQUATIONS}

Evolution of the beam longitudinal distribution function is described by:

$$
\frac{\partial f}{\partial t}+\frac{\partial}{\partial x}(F(x) f)=\frac{1}{2} \frac{\partial}{\partial x}\left(D(x) \frac{\partial f}{\partial x}\right)
$$

The drag force is created by the particle self-interaction and therefore is not directly affected by the band overlap but is affected by screening of the particle signal. The result is well-known ${ }^{[1]}$ :

$$
F(x) \equiv \frac{d x}{d t}=\frac{1}{T} \sum_{n=-\infty}^{\infty} \frac{G_{1}\left(x, \omega_{n}\right)}{\varepsilon\left(\omega_{n}\right)}\left(1-A\left(\omega_{n}\right) e^{-i \omega_{n} T_{0}}\right) e^{i \omega_{n} T_{2} \eta_{2} x}, \quad \omega_{n}=\omega_{0}(1+\eta x) n .
$$

Here $G_{1}(x, \omega)=G(x, \omega) / N$ is the single particle gain, $N$ is the particle number in the beam and $\varepsilon\left(\omega_{n}\right)$ in the denominator takes into account particle screening ${ }^{[3]}$.

The diffusion is created by noise in the kicker voltage:

$$
D(x)=\frac{2 \pi e^{2}}{T_{0}^{2}\left(\gamma \beta^{2} m c^{2}\right)^{2}} \sum_{n=-\infty}^{\infty} P_{U}\left(\omega_{n}\right),
$$

where $P_{U}(\omega)$ is the spectral density of kicker voltage consisting of two contributions. The first one is related to the noise of the electronics, $P_{\text {Unoise }}$, and the second one is related to the particle shot noise. Note that we normalize all spectral densities so that $\int_{-\infty}^{\infty} P_{x}(\omega) d \omega=\overline{x^{2}}$. The beam current shot noise for non-interacting particles is equal to:

$$
P_{I}(\omega)=\frac{e^{2} N}{2 \pi T_{0}} \sum_{k=-\infty}^{\infty} \frac{1}{|k \eta|} f\left(\frac{\omega-k \omega_{0}}{\eta k \omega_{0}}\right) .
$$

Combining Eqs. (27) and (28) and simplifying one obtains:

$$
D(x)=\sum_{n=-\infty}^{\infty}\left[\frac{2 \pi e^{2} P_{\text {Unoise }}\left(\omega_{n}\right)}{T_{0}^{2}\left(\gamma \beta^{2} m c^{2}\right)^{2}}+\frac{N}{T_{0}}\left|\frac{G_{1}\left(x, \omega_{n}\right)\left(1-A\left(\omega_{n}\right) e^{-i \omega_{n} T_{0}}\right)}{\varepsilon\left(\omega_{n}\right)}\right|^{2} \sum_{k=-\infty}^{\infty} \frac{1}{|k \eta|} f\left(\frac{(1+\eta x) n-k}{\eta k}\right)\right] .
$$

Natural variables for transverse cooling description are the action-phase variables $(I, \psi)$. We determine the action so that $I=\left(\beta_{y} \theta^{2}+2 \alpha_{y} y \theta+\left(1+\alpha_{y}{ }^{2}\right) y^{2} / \beta_{y}\right) / 2$, where $\beta_{y}$ and $\alpha_{y}$ are the beta- and alpha-functions of the ring. We assume that there is no $x-y$ coupling in the lattice, and the cooling is linear in betatron amplitude. That yields that 
the beam distribution function can be described by the following equation:

$$
\frac{\partial f_{\perp}}{\partial t}+\lambda_{\perp}(x) \frac{\partial}{\partial I}\left(I f_{\perp}\right)=D_{\perp}(x) \frac{\partial}{\partial I}\left(I \frac{\partial f_{\perp}}{\partial I}\right) \quad .
$$

Here $f_{\perp} \equiv f_{\perp}(x, I, t)$ is the distribution function normalized so that $\int f_{\perp}(x, I, t) d I=f_{0}(x)$ and the same as above $\int f_{0}(x) d x=1, \lambda_{\perp}(x)$ is the cooling decrement, and $D_{\perp}(x)$ is the diffusion coefficient. $\lambda_{\perp}(x)$ and $D_{\perp}(x)$ do not depend on $I$ because of system linearity in the transverse plane.

Similar to the longitudinal cooling the transverse cooling is created by the particle self-interaction and therefore is not directly affected by the band overlap but still affected by screening. The result is ${ }^{[1]}$ :

$$
\lambda_{\perp}(x)=\frac{1}{T_{0}} \sum_{n=-\infty}^{\infty} \operatorname{Re}\left(i \frac{G_{\perp 1}\left(\omega_{n}\right)}{\varepsilon_{\perp}\left(\omega_{n}\right)} e^{i \omega_{n} T_{2} \eta_{2} x-2 \pi i \nu_{2}}\right), \quad \omega_{n}=\omega_{0}(n(1+\eta x)-(v+\xi x)),
$$

where $G_{\perp 1}(\omega)=G_{\perp}(\omega) / N$ is the single particle gain.

The diffusion coefficient is:

$$
D_{\perp}(x)=\frac{\pi \beta_{k}}{2 T_{0}^{2}} \sum_{n=-\infty}^{\infty} P_{\theta}\left(\omega_{n}\right),
$$

where $P_{\theta}(\omega)$ is the spectral density of the angle kicks produced by the kicker. $P_{\theta}(\omega)$ consists of two contributions: the spectral density of amplifier noise, $P_{\perp U a}(\omega)$, and the amplified shot noise of the beam. The shot noise of the beam at the pickup is

$$
P_{\perp U_{p}}(\omega)=\frac{e^{2} \mid Z_{p \perp}(\omega)^{2} \overline{y^{2}}}{T_{0}^{2}} N \sum_{m=-\infty}^{\infty} \frac{1}{\left\|\omega_{0}(\xi+\eta m)\right\|} f\left(\frac{\omega-\omega_{0}(v+m)}{\omega_{0}(\xi+\eta m)}\right),
$$

Substituting Eq. (33) into Eq. (32) and using definition of the single particle gain we obtain:

$$
D_{\perp}(x)=\sum_{n=-\infty}^{\infty}\left(\frac{\pi \beta_{k}}{2 T_{0}^{2}}\left(\frac{e\left|Z_{k \perp}\left(\omega_{n}\right)\right|}{m c^{2} \beta^{2} \gamma Z_{\text {ampl }}}\right)^{2} P_{\perp U}\left(\omega_{n}\right)+\left|\frac{G_{\perp 1}\left(\omega_{n}\right)}{\varepsilon_{\perp}\left(\omega_{n}\right)}\right|^{2} \overline{\frac{I(x)}{}} \frac{1}{2 T_{0}} \sum_{m=-\infty}^{\infty} \frac{f\left(\frac{n-m+(\xi+\eta m) x}{\xi+\eta m}\right)}{|\xi+\eta m|}\right),
$$

where $\overline{I(x)}=\int f_{\perp}(x, I, t) I d I$ is the average action for given momentum deviation $x$.

\section{ACKNOWLEDGMENTS}

The author would like to thank A. Burov and J. Bisognano for many fruitful discussions.

\section{REFERENCES}

1. J. Bisognano and C. Leemann, "Stochastic Cooling" in 1981 Summer School on High Energy Particle Accelerators, edited by R. A. Carrigan et al., AIP Conference Proceedings 87, American Institute of Physics, Melville, NY, 1982, pp. 584-655.

2. D. Möhl, "Stochastic Cooling" in CERN Accelerator School, Fifth Advanced Accelerator Physics Course, edited by S. Turner, CERN, Geneva, Switzerland, 1995, pp. 587-671.

3. V. V. Parkhomchuk and D. V. Pestrikov, Sov. Phys. Tech. Phys, 25(7), 818 (1980). 